\title{
EXTENDING STECHKIN'S THEOREM AND BEYOND
}

\author{
TUDOR ZAMFIRESCU
}

Received 24 November 2003

We generalize Stechkin's theorem about the single-valuedness of the nearest point mapping at most points to any complete length space without bifurcating geodesics and make further observations.

Let $K \subset \mathbb{R}^{d}$ be a closed set and $p_{K}$ the nearest point mapping, which associates with every point $x \in \mathbb{R}^{d}$ the set of all points in $K$ closest to $x$. It is well known that $p_{K}$ is single-valued a.e. The topological counterpart was provided by Stechkin [6] in 1963, who proved that $p_{K}$ is single-valued at most points of $\mathbb{R}^{d}$, that is, the set of points at which $p_{K}$ is not single-valued is of the first Baire category.

A result attributed to Konjagin says that the set of points where $p_{K}$ is not single-valued is even $\sigma$-porous (see also [7]). This implies both statements, on measure and category.

We showed in [8] that, in any Alexandrov space with curvature bounded below, $p_{K}$ is properly multivalued on a $\sigma$-porous set.

We extend here Stechkin's result to more general metric spaces, and provide related results under even more general assumptions.

Let $(X, \rho)$ be a metric space and let $K \subset X$ be compact. As usual, for $x \in X$,

$$
p_{K}(x)=\{y \in K: \rho(x, y)=\rho(x, K)\},
$$

where $\rho(x, K)=\min _{z \in K} \rho(x, z)$.

The open ball of centre $x$ and radius $\varepsilon$ will be denoted by $B(x, \varepsilon)$. We will also use the notation

$$
A_{n}=\left\{x \in X: \operatorname{diam} p_{K}(x) \geq \frac{1}{n}\right\} .
$$

Let $\mathscr{K}$ be the space of all nonempty compact subsets of $X$ and $H$ the Pompeiu-Hausdorff distance in $\mathscr{K}$, that is,

$$
H\left(K, K^{\prime}\right)=\max \left\{\max _{a \in K} \rho\left(a, K^{\prime}\right), \max _{b \in K^{\prime}} \rho(b, K)\right\} .
$$

The space $(\mathscr{K}, H)$ is complete as soon as $(X, \rho)$ is itself complete. 
An $\operatorname{arc} \sigma$ in $X$ from $x$ to $y$ is called a segment if its length $\lambda \sigma$ equals $\rho(x, y)$. A metric space (with more than one point) in which there is a segment between any two points is called a length space.

We say that a metric space is without bifurcating geodesics if, for any two segments starting at the same point and having another common point, this second point is a common endpoint of both or one segment includes the other.

We start with the following generalization of Stechkin's theorem.

Theorem 1 . For any compact set $K$ in the complete length space $(X, \rho)$ without bifurcating geodesics, $p_{K}$ is single-valued at most points $x \in X$.

Proof. Suppose the conclusion of the theorem is false. Then $p_{K}$ is not single-valued on a set of second category. This means that $\bigcup_{n=1}^{\infty} A_{n}$ is of second category. Hence, for some index $n, A_{n}$ is dense in a ball $B(a, r)$.

Let $y \in p_{K}(a)$. Consider a segment ay and some point $x \in a y \cap B(a, r)$. Let $y^{\prime} \in p_{K}(x)$. Since $\rho\left(a, y^{\prime}\right) \geq \rho(a, y)$ and

$$
\rho\left(a, y^{\prime}\right) \leq \rho(a, x)+\rho\left(x, y^{\prime}\right) \leq \rho(a, y)
$$

we must have the equality sign, and the segments $a y$ and $a x \cup x y^{\prime}$ would bifurcate if $y \neq y^{\prime}$. It follows that $p_{K}(x)=\{y\}$.

Since $p_{K}$ is upper semicontinuous, there is some neighbourhood $N$ of $x$ such that $p_{K}(u) \subset B(y, 1 /(3 n))$ for all $u \in N$.

Then diam $p_{K}(u)<1 / n$ for all these $u, A_{n}$ is not dense in $B(a, r)$, and a contradiction is obtained.

Hence the conclusion of the theorem is true.

Theorem 1 shows that $p_{K}$ is single-valued at many points. It is, however, rarely singlevalued everywhere.

THeOREM 2. In any length space $(X, \rho), p_{K}$ is single-valued only for a nowhere dense family of sets $K \in \mathcal{K}$.

Proof. Let $O \subset \mathcal{K}$ be open and assume $p_{K}$ is single-valued for some set $K \in \mathcal{O}$. If $K \neq X$, take $x \in X \backslash K$, put $\left\{z_{2}\right\}=p_{K}(x)$, and take $z_{1} \in x z_{2}$ at distance $\varepsilon$ from $z_{2}$ such that $K^{\prime}=$ $K \cup\left\{z_{1}\right\} \in \mathcal{O}$. If $K=X$, take a segment $\sigma \subset X$, choose its midpoint $z$, and consider a positive number $\varepsilon \leq \lambda \sigma$ such that every $K^{*} \in \mathcal{K}$ with $H\left(K^{*}, X\right)<\varepsilon$ belongs to 0 . Now let $z_{1}, z_{2}$ denote the two points of $\sigma$ at distance $\varepsilon / 2$ from $z$ and define

$$
K^{\prime}=(X \backslash B(z, \varepsilon)) \cup\left\{z_{1}, z_{2}\right\}
$$

Clearly, $K^{\prime} \in O^{\text {. }}$

If $H\left(K^{\prime}, K^{\prime \prime}\right)<\varepsilon / 4, p_{K^{\prime \prime}}$ is not single-valued. Indeed, there are points $u_{1}, u_{2} \in K^{\prime \prime}$ with $\rho\left(u_{i}, z_{i}\right)<\varepsilon / 4$. Of course, $p_{K^{\prime \prime}}\left(z_{i}\right) \in B\left(z_{i}, \varepsilon / 4\right)$ for both $i$. Also, for every point $x$ on the segment $z_{1} z_{2}$,

$$
p_{K^{\prime \prime}}(x) \in B\left(z_{1}, \frac{\varepsilon}{4}\right) \cup B\left(z_{2}, \frac{\varepsilon}{4}\right)
$$


It follows that there must be a point $u \in z_{1} z_{2}$ with $p_{K^{\prime \prime}}(u)$ properly multivalued. Since $\left\{K^{\prime \prime}: H\left(K^{\prime}, K^{\prime \prime}\right)<\varepsilon / 4\right\}$ is open in $\mathscr{K}$, the theorem is proven.

We presented in [8, Theorem 3] a result on most compact sets in an Alexandrov space of curvature bounded below. Since the only property exploited in its proof is the absence of bifurcating geodesics, we can formulate the following theorem and refer to the proof in [8].

Theorem 3. In a complete separable length space $(X, \rho)$ without bifurcating geodesics, in which every open set has Hausdorff dimension larger than 1 , for most $K \in \mathscr{K}, p_{K}$ is properly multivalued at a dense set of points.

Already [8, Theorem 3] is a generalization of an old result of the author [7], which had been repeatedly strengthened in various ways, for example, in $[1,2,3,4,5,9]$.

Without the condition about the nonexistence of bifurcating geodesics, we can only prove the following theorem.

Theorem 4. For most compact sets $K$ in the complete separable length space $(X, \rho), p_{K}$ is single-valued at most points $x \in X$.

Proof. Fix an open ball $B(a, r) \subset X$. We show that the set $\mathcal{K}_{n}$ of all compact sets $K \subset X$ for which $\left\{x \in X: \operatorname{diam} p_{K}(x) \geq 1 / n\right\}$ is dense in $B(a, r)$ is nowhere dense in $\mathscr{K}$.

Let $O \subset \mathcal{K}$ be open. We choose $K \in \mathcal{O}$ such that $a \notin K$.

Let $y \in p_{K}(a)$. Choose a segment $a y$ and a point $z \in a y$ such that $K \cup\{z\} \in \mathbb{O}$. Take

$$
\varepsilon=\min \left\{\frac{\rho(y, z)}{4}, \frac{1}{2 n}\right\}
$$

Then, for every point $x \in B(a, \varepsilon)$, every point $v$ at distance at most $\varepsilon$ from $K$ and every point $w \in B(z, \varepsilon)$, we have

$$
\begin{aligned}
& \rho(x, v)>\rho(a, y)-2 \varepsilon, \\
& \rho(x, w)<\rho(a, z)+2 \varepsilon .
\end{aligned}
$$

Hence, for every point $x \in B(a, \varepsilon)$ and every compact set $K^{\prime}$ at Pompeiu-Hausdorff distance at most $\varepsilon$ from $K \cup\{z\}$,

$$
p_{K^{\prime}}(x) \subset B(z, \varepsilon)
$$

whence

$$
\operatorname{diam} p_{K^{\prime}}(x)<\frac{1}{n}
$$

Let $K$ be such that $p_{K}$ is not single-valued on a set of second category. Then

$$
\left\{x \in X: \operatorname{diam} p_{K}(x)>0\right\}=\bigcup_{n=1}^{\infty} A_{n}
$$

is of second category. This implies that, for some $n$, the set $A_{n}$ is dense in some ball. 
Since $X$ is separable, there is a countable set $\left\{x_{i}\right\}_{i=1}^{\infty}$ dense in $X$. Hence $A_{n}$ is dense in some ball $B\left(x_{i}, r_{j}\right)$ with rational radius $r_{j}$.

Hence all compact sets $K$ for which $p_{K}$ is not single-valued on a set of second category belongs to the union $\bigcup_{n, i, j=1}^{\infty} \mathcal{K}_{n, i, j}$, where $\mathcal{K}_{n, i, j}$ is the set of those $K \in \mathscr{K}$ for which $A_{n}$ is dense in $B\left(x_{i}, r_{j}\right)$.

Since we showed that each $\mathscr{K}_{n, i, j}$ is nowhere dense, the union above is of first category and the theorem is proven.

\section{References}

[1] F. S. De Blasi, P. S. Kenderov, and J. Myjak, Ambiguous loci of the metric projection onto compact starshaped sets in a Banach space, Monatsh. Math. 119 (1995), no. 1-2, 23-36.

[2] F. S. De Blasi and J. Myjak, Ambiguous loci in best approximation theory, Approximation Theory, Spline Functions and Applications (Maratea, 1991) (S. P. Singh, ed.), NATO Adv. Sci. Inst. Ser. C Math. Phys. Sci., vol. 356, Kluwer Academic, Dordrecht, 1992, pp. 341-349.

[3] Ambiguous loci of the nearest point mapping in Banach spaces, Arch. Math. (Basel) 61 (1993), no. 4, 377-384.

[4] On compact connected sets in Banach spaces, Proc. Amer. Math. Soc. 124 (1996), no. 8, 2331-2336.

[5] F. S. De Blasi and T. I. Zamfirescu, Cardinality of the metric projection on typical compact sets in Hilbert spaces, Math. Proc. Cambridge Philos. Soc. 126 (1999), no. 1, 37-44.

[6] S. Stechkin, Approximative properties of subsets of Banach spaces, Rev. Roumaine Math. Pures Appl. 8 (1963), 5-8 (Russian).

[7] T. Zamfirescu, The nearest point mapping is single valued nearly everywhere, Arch. Math. (Basel) 54 (1990), no. 6, 563-566.

[8] On the cut locus in Alexandrov spaces and applications to convex surfaces, Pacific J. Math. 217 (2004), no. 2, 375-386.

[9] N. V. Zhivkov, Compacta with dense ambiguous loci of metric projections and antiprojections, Proc. Amer. Math. Soc. 123 (1995), no. 11, 3403-3411.

Tudor Zamfirescu: Department of Mathematics, University of Dortmund, 44221 Dortmund, Germany

E-mail address: tudor.zamfirescu@mathematik.uni-dortmund.de 


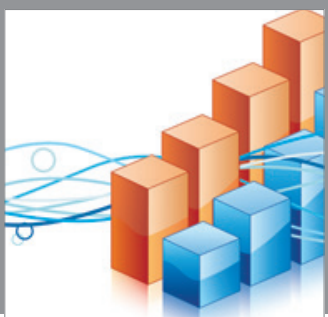

Advances in

Operations Research

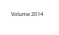

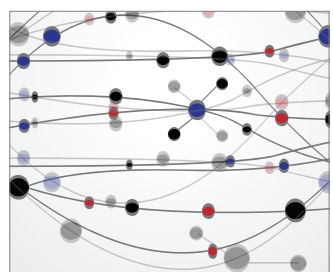

\section{The Scientific} World Journal
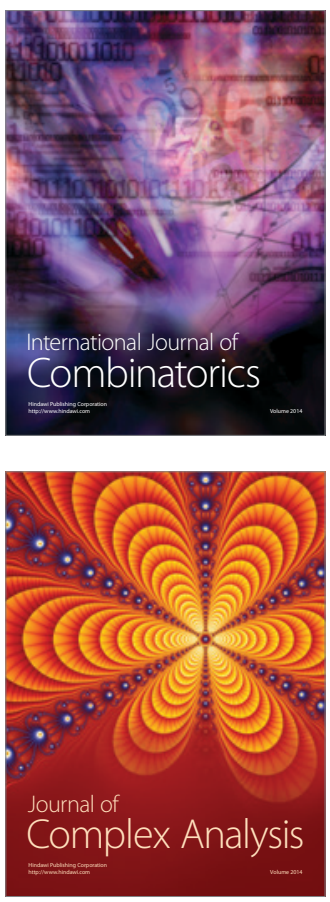

International Journal of

Mathematics and

Mathematical

Sciences
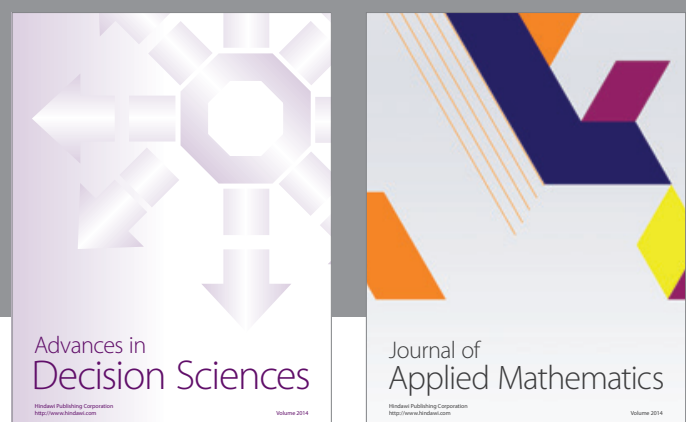

Journal of

Applied Mathematics
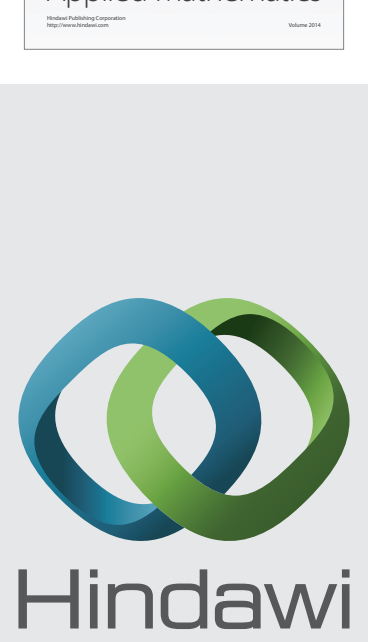

Submit your manuscripts at http://www.hindawi.com
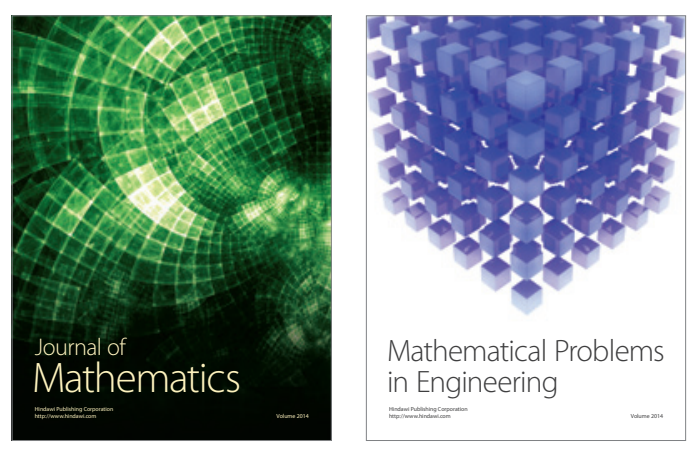

Mathematical Problems in Engineering
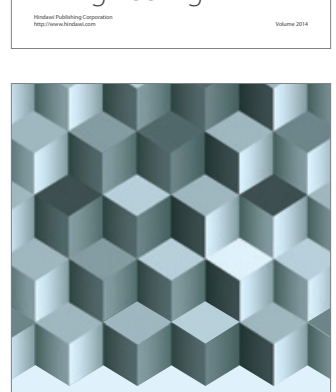

Journal of

Function Spaces
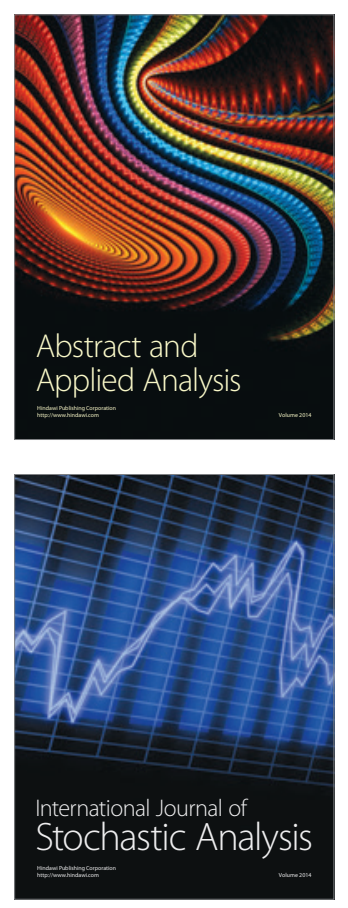

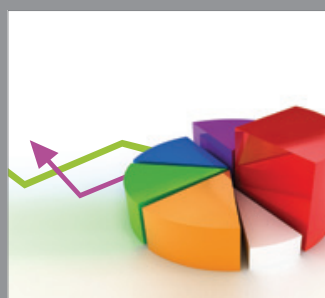

ournal of

Probability and Statistics

Promensencen
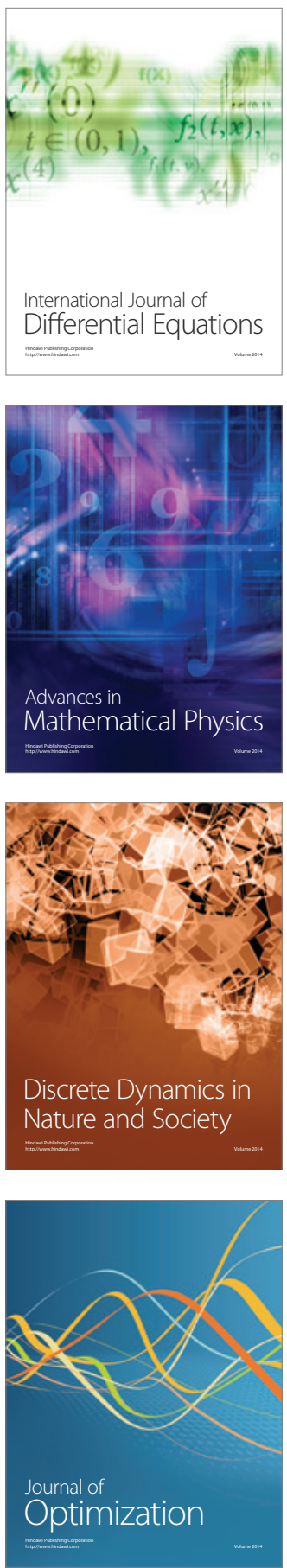